\title{
In-Stent Restenosis due to Stent Recoil After Third- Generation Drug-Eluting Stent Implantation
}

\author{
Yuta Kato ${ }^{\mathrm{a}}$, Atsushi Iwata ${ }^{\mathrm{a}, \mathrm{b}}$, Masayuki Nakamura ${ }^{\mathrm{a}}$, Shin-ichiro Miura ${ }^{\mathrm{a}}$, \\ Keijiro Saku ${ }^{\mathrm{a}}$
}

\begin{abstract}
A 72-year-old man with a heavily calcified coronary lesion underwent percutaneous coronary intervention (PCI) using a third-generation drug-eluting stent (DES) with thin stent struts. Two months after the PCI, in-stent restenosis due to stent recoil (SR) occurred. An intravascular ultrasound (IVUS) revealed deformed stent struts and heavily calcified coronary plaque. The SR probably occurred due to persistent vessel compression as a result of heavily calcified coronary plaque. We should consider using a DES with thick stent struts rather than a third-generation DES with thin stent struts in patients with a heavily calcified coronary lesion.
\end{abstract}

Keywords: Stent recoil; In-stent restenosis; Drug-eluting stent; Calcified coronary plaque

\section{Introduction}

Percutaneous coronary intervention (PCI) using drug-eluting stents (DESs) is a standard treatment for coronary artery disease. New-generation DESs incorporate a durable and flexible structure, biocompatible polymers and optimized drugs to reduce adverse cardiovascular events such as in-stent restenosis (ISR) and late stent thrombosis. Recently, third-generation DESs, which have thin stent struts and are based on biodegradable polymer technology, are developed to provide polymer-free surroundings and to reduce both vascular injury and persistent inflammatory stimulation at the stented segment. Although third-generation DESs have been reported to give an excellent clinical outcome [1], thin stent struts could reduce their radial strength and lead to an increased risk of stent deformation. An abnormal late-acquired stent axial deformation,

Manuscript accepted for publication April 10, 2017

aDepartment of Cardiology, Fukuoka University School of Medicine, Fukuoka, Japan

${ }^{b}$ Corresponding Author: Atsushi Iwata, Department of Cardiology, Fukuoka University School of Medicine, 7-45-1 Nanakuma Jonan-ku, Fukuoka 8140180, Japan. Email: iwaiwa@fukuoka-u.ac.jp

doi: https://doi.org/10.14740/jocmr3024w so-called stent recoil (SR), is rarely observed after coronary stent implantation $[2,3]$. We describe a case of ISR due to SR after third-generation DES implantation.

\section{Case Report}

A 72-year-old man who had a history of diabetes mellitus, hypertension and dyslipidemia was referred to our hospital due to worsening effort angina. Electrocardiogram (ECG) and chest $\mathrm{X}$-ray were normal, and cardiac enzymes including high-sensitivity troponin I were not significantly increased. Coronary angiogram (CAG) showed moderate-severe stenosis in the proximal-mid portion of the left anterior descending coronary artery (LAD) (Fig. 1a). PCI under intravascular ultrasound (IVUS) guidance was attempted; however, the IVUS catheter (Terumo Corp. Tokyo, Japan) did not pass the target lesion. Therefore, rotational atherectomy (RA) using a $1.5-\mathrm{mm}$ burr (Boston Scientific Corp., MA, USA) was performed (Fig. 1b). After RA, pre-dilation with a $2.5 / 15 \mathrm{~mm}$ non-compliant balloon at 20 atm was performed (Fig. 1c), and a 2.5/38 mm Synergy stent (Boston Scientific Corp., MA, USA), a third-generation DES, was implanted in the target lesion. After post-dilation using the 2.5/15 $\mathrm{mm}$ non-compliant balloon at high pressure (22 atm), optimal angiographic and IVUS results were obtained (Figs. $1 \mathrm{~d}$ and $2 \mathrm{a}, \mathrm{b})$.

Two months later, he was admitted again due to the recurrence of chest pain. Although the ECG and biochemical analysis were normal, CAG showed focal ISR in the mid portion of the LAD (Fig. 3a). IVUS revealed deformed stent struts without obvious separation of the structure and heavily calcified plaque with a minimum lumen area of $1.9 \mathrm{~mm}^{2}$ (Fig. 2c, d). The cause of ISR was considered to be focal SR, which probably occurred due to persistent vessel compression as a result of heavily calcified coronary plaque. We decided to ablate using RA to eliminate the heavily calcified plaque as much as possible together with the deformed stent struts. RA was performed cautiously using 1.75 - and 2.0 -mm burrs (Fig. $3 \mathrm{~b}$ ) according to a stepped burr approach reported previously [4]. After RA and high-pressure dilatation (22 atm) using a 2.5/15 $\mathrm{mm}$ non-compliant balloon (Fig. 3c), CAG showed an optimal result (Fig. 3d). IVUS showed that part of the stent struts had disappeared by RA and adequate lesion expansion was obtained without placement of an additional stent (Fig. 2e, f). Complications such as no reflow/slow flow phenomenon or an 


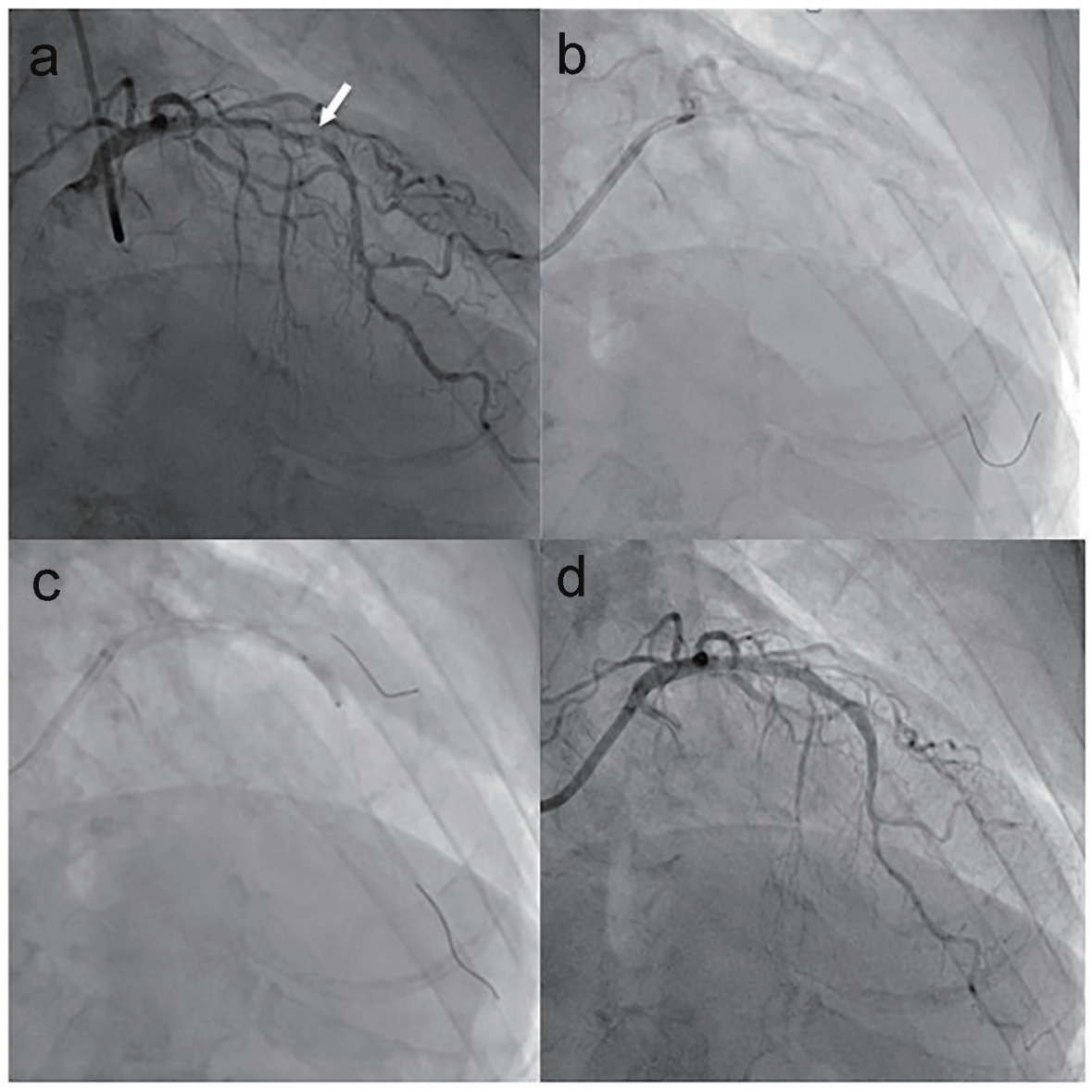

Figure 1. CAG images of the first PCI. (a) Coronary stenosis in the proximal-mid portion of LAD with a severe focal stenosis (arrow). (b) RA with a 1.5-mm burr. (c) Pre-dilation with a 2.5-mm non-compliant balloon. (d) After DES implantation. CAG: coronary angiogram; PCl: percutaneous coronary intervention; LAD: left anterior descending coronary artery; RA: rotational atherectomy; DES: drug-eluting stent.

increase in cardiac enzymes were not found after PCI.

However, focal recurrent ISR occurred 2 months later. Therefore, a $2.5 / 8 \mathrm{~mm}$ Nobori stent (Terumo Corp., Tokyo, Japan), which is a second-generation DES that has thicker stent struts than a Synergy stent, was deployed followed by highpressure (22 atm) post-dilation with a $2.5 / 15 \mathrm{~mm}$ non-compliant balloon. Furthermore, a third ISR occurred 2 months later, and IVUS again detected focal SR at the ISR site. Therefore, an additional $2.5 / 8 \mathrm{~mm}$ Nobori stent implantation and post-dilation with a $2.5 / 12 \mathrm{~mm}$ non-compliant balloon at $24 \mathrm{~atm}$ was conducted. Finally, two layers of DES with thick stent struts were implanted at the ISR site, and follow-up CAG did not document ISR after the final PCI (Fig. 3e).

\section{Discussion}

So-called SR, an abnormal late-acquired stent axial deformation, rarely occurs after coronary stent implantation. A recent study using IVUS reported that the incidence of focal SR after bare-metal stent or first-generation DES implantation was $1.8 \%$ [3]. In a more recent study, the incidence of SR was $1.2 \%$ in patients who received first- or second-generation DES implantation [2]. Although the incidence of SR after coronary stent placement is low, if it does occur, SR often leads to ISR [5]. To our knowledge, this is the first case report of ISR caused by focal SR after third-generation DES implantation. Focal SR is defined as a more than $20 \%$ decrease in the minimum stent area as assessed by IVUS [3], and the IVUS finding of focal SR in this case obviously met this criterion (Fig. 2c, d).

The RA procedure in the first PCI for the heavily calcified lesion was appropriately performed according to a previous report [6]; a $1.5 \mathrm{~mm}$ burr of RA was used and the burr-to-artery ratio was 0.6 (Fig. 1b). As shown in Fig. 2a, b, the IVUS images immediately after the first PCI showed optimal stent expansion. Despite the appropriate procedure and optimal result, early ISR due to focal SR occurred 2 months after the first PCI (Figs. 2c, d and 3a).

Thin stent struts can minimize vascular injury and subsequent neo-intimal hyperplasia [7], and stent deliverability to a target lesion could be improved by reducing the stent strut thickness [8]. Therefore, the struts of the third-generation DES Synergy stent are thinner (74- $\mu \mathrm{m}$ thick) than those of previousgeneration stents including second-generation DES (more than 

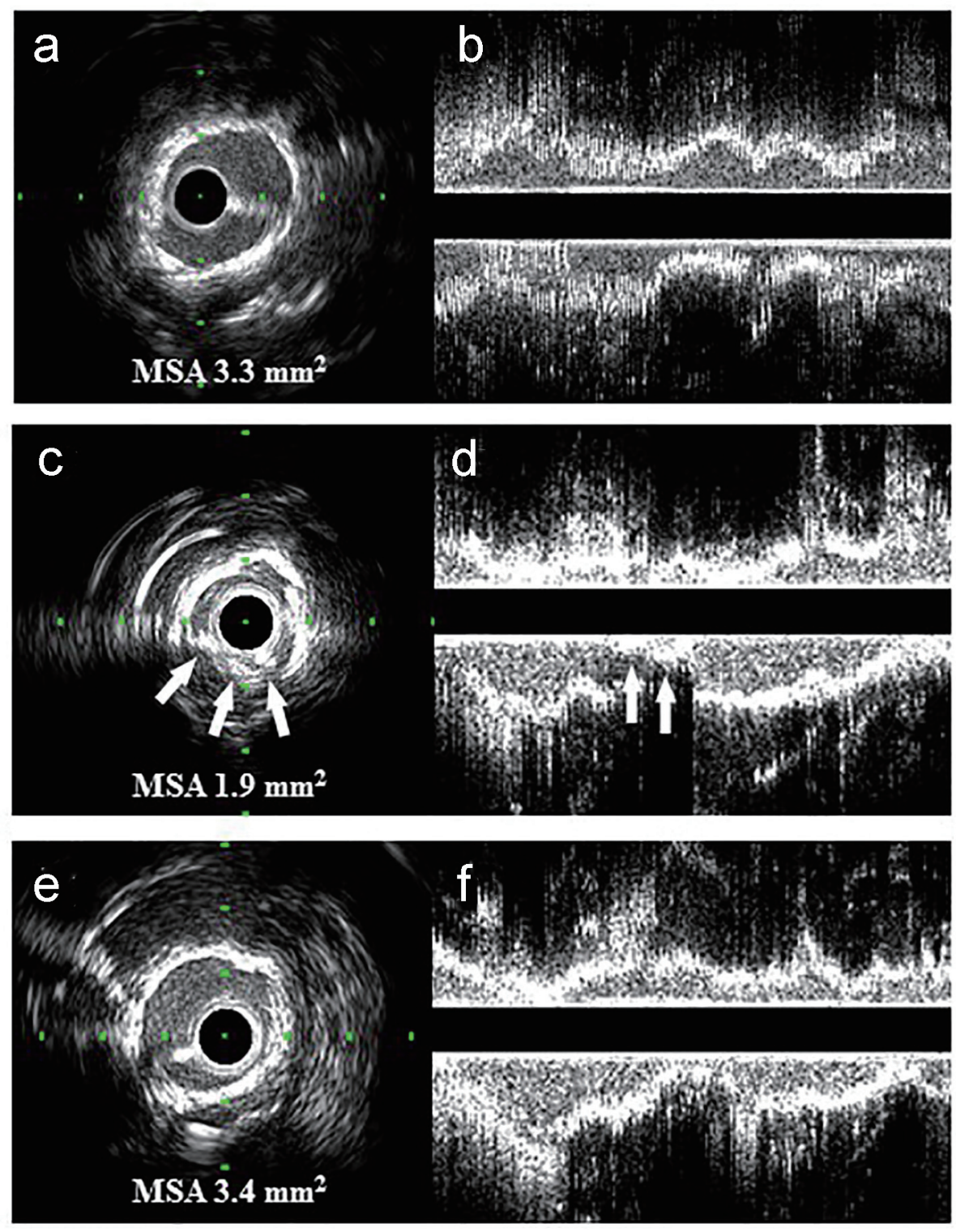

Figure 2. Transversal (a, c and e) and longitudinal (b, $d$ and f) IVUS images of this case: $(a, b)$ immediately after the first PCl; $(c, d)$ the deformed stent struts (arrows) and heavily calcified coronary plaque at the ISR site with an MSA of $1.9 \mathrm{~mm}^{2} ;(\mathrm{e}, \mathrm{f})$ wellexpanded ISR site and disappearance of part of the stent struts. IVUS: intravascular ultrasound; PCI: percutaneous coronary intervention; ISR: in-stent restenosis; MSA: minimum stent area.

80- $\mu \mathrm{m}$ thick) to improve clinical outcomes after PCI. In fact, the Synergy stent promoted faster stent-strut neo-intimal coverage and reduced the incidence of neo-atherosclerosis compared to a second-generation DES in an atherosclerotic rabbit model [9], and has been associated with an excellent clinical outcome [1]. However, thin stent struts may reduce its radial strength and increase the incidence of SR [8]. Ohya et al reported that calcified plaque at the PCI site was an independent predictor of SR [2]. Heavily calcified coronary plaque could persistently compress stent struts and cause metal fatigue, and may increase the risk of SR. Therefore, a third-generation DES with thin stent struts may not be suitable for the treatment of heavily calcified coronary lesions, and a DES with thicker stent struts and stronger radial force probably should have been used in this case.

In the second PCI, we eliminated part of the heavily calcified plaque and the deformed stent struts using RA with a 2.0$\mathrm{mm}$ burr, and dilated the ISR site using a non-compliant balloon with high pressure (Fig. 3b, c). As a result, optimal lumen expansion was obtained (Figs. 2e, f and Fig. 3d) without the need for additional stent implantation as previously reported [10]. However, recurrent ISR occurred. Therefore, we implanted another DES Nobori stent with thicker stent struts $(125-\mu \mathrm{m}$ thick). Finally, two layers of Nobori stents were implanted in the ISR site, and follow-up CAG demonstrated a satisfactory result (Fig. 3e). Williams et al reported that double-stenting 


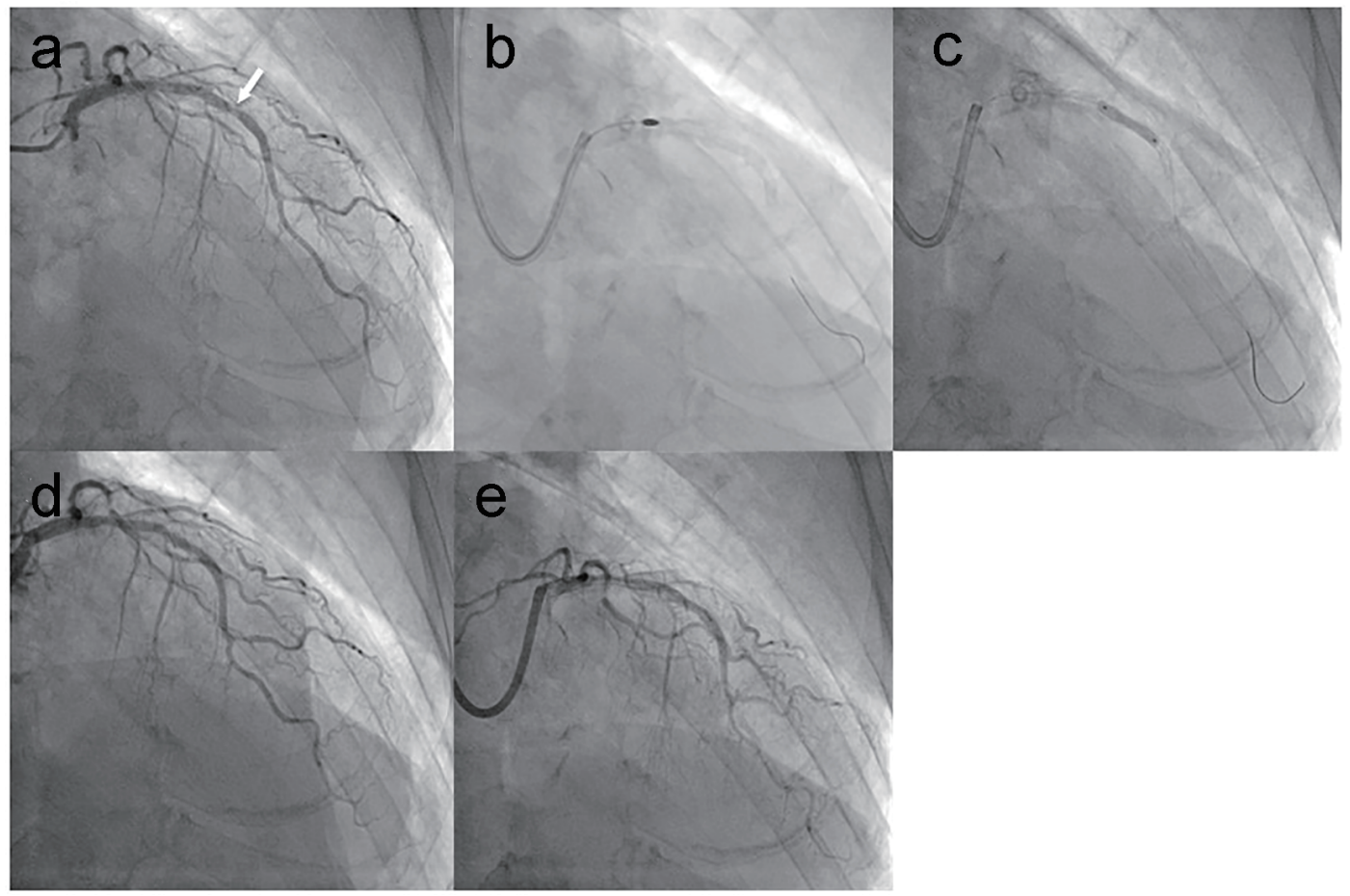

Figure 3. CAG images at 2 months after the first $\mathrm{PCl}$ and at follow-up after the final PCl: (a) focal ISR in the mid portion of the LAD (arrow); (b) RA with a 2.0-mm burr; (c) high-pressure dilation with a 2.5-mm non-compliant balloon; (d) immediately after the second PCl; (e) at follow-up after the final PCl. CAG: coronary angiogram; PCl: percutaneous coronary intervention; ISR: in-stent restenosis; LAD: left anterior descending coronary artery; RA: rotational atherectomy.

can significantly improve the angiographic outcome of acute stent recoil [11]. Despite aggressive RA using a 2.0-mm burr (burr-to-artery ratio 0.8 ) and high-pressure non-compliant balloon dilation in the second PCI (Fig. 3b, c), focal SR and ISR occurred again in this case. Double-stenting using a secondgeneration DES with thick stent struts may be an effective strategy in patients with ISR due to SR and a severely calcified coronary lesion, as in this case.

We experienced a rare case of ISR due to focal SR after third-generation DES implantation. In patients with a heavily calcified coronary lesion, we should consider using a DES with thick stent struts rather than a third-generation DES with thin stent struts.

\section{Disclosures}

Keijiro Saku (KS) and Shin-ichiro Miura (SM) are Directors of NPO Clinical and Applied Science, Fukuoka, Japan. KS and SM had received a grant from the Public Interest Incorporated Foundation of "Clinical Research Promotion Foundation" in Fukuoka, Japan, and part of this work was transferred to NPO Clinical and Applied Science, Fukuoka, Japan. KS has an Endowed Department of Molecular Cardiovascular Therapeutics (SM), Fukuoka University, supported by MSD Co., Ltd, and an Endowed Department of Community and Emergency Medicine (MS, HN), Fukuoka University, supported by Izumi City, Kagoshima, Japan.

\section{References}

1. Meredith IT, Verheye S, Dubois CL, Dens J, Fajadet J, Carrie D, Walsh S, et al. Primary endpoint results of the EVOLVE trial: a randomized evaluation of a novel bioabsorbable polymer-coated, everolimus-eluting coronary stent. J Am Coll Cardiol. 2012;59(15):1362-1370.

2. Ohya M, Kadota K, Kubo S, Tada T, Habara S, Shimada T, Amano H, et al. Incidence, predictive factors, and clinical impact of stent recoil in stent fracture lesion after drugeluting stent implantation. Int J Cardiol. 2016;214:123129.

3. Koo BK, Waseda K, Ako J, Hasegawa T, Shimohama T, Nakatani D, Otake H, et al. Incidence of diffuse and focal chronic stent recoil after implantation of current generation bare-metal and drug-eluting stents. Int J Cardiol. 2010;144(1):132-134.

4. Kobayashi Y, Teirstein P, Linnemeier T, Stone G, Leon M, Moses J. Rotational atherectomy (stentablation) in a lesion with stent underexpansion due to heavily calcified plaque. Catheter Cardiovasc Interv. 2001;52(2):208-211.

5. Hong MK, Park SW, Lee CW, Ko JY, Kang DH, Song JK, Kim JJ, et al. Intravascular ultrasound comparison of chronic recoil among different stent designs. Am J Cardiol. 1999;84(10):1247-1250, A1248.

6. Tomey MI, Kini AS, Sharma SK. Current status of rotational atherectomy. JACC Cardiovasc Interv. 2014;7(4):345-353. 
7. Kastrati A, Mehilli J, Dirschinger J, Dotzer F, Schuhlen $\mathrm{H}$, Neumann FJ, Fleckenstein $\mathrm{M}$, et al. Intracoronary stenting and angiographic results: strut thickness effect on restenosis outcome (ISAR-STEREO) trial. Circulation. 2001;103(23):2816-2821.

8. Menown IB, Noad R, Garcia EJ, Meredith I. The platinum chromium element stent platform: from alloy, to design, to clinical practice. Adv Ther. 2010;27(3):129141.

9. Nakazawa G, Torii S, Ijichi T, Nagamatsu H, Ohno Y, Kurata $\mathrm{F}$, Yoshikawa A, et al. Comparison of Vascular Re- sponses Following New-Generation Biodegradable and Durable Polymer-Based Drug-Eluting Stent Implantation in an Atherosclerotic Rabbit Iliac Artery Model. J Am Heart Assoc. 2016;5(10).

10. Frisoli TM, Friedman H, O'Neill WW. Rotational Atherectomy of Three Overlapping Stent Layers. J Invasive Cardiol. 2016;28(9):E77-79.

11. Williams PD, Appleby CE, Chowdhary S, Fraser DG. Double stenting: a method for treating acute stent recoil and luminal filling defects. EuroIntervention. 2011;6(7):846-853. 